\title{
Influence of prolonged translaryngeal intubation on airway complications: a retrospective comparative analysis
}

\author{
Takeru Shimizu $^{1}$ D $\cdot$ Taro Mizutani $^{2} \cdot$ Keiichi Hagiya $^{3} \cdot$ Makoto Tanaka $^{1}$
}

Received: 14 April 2019 / Accepted: 24 May 2019 / Published online: 31 May 2019

c) Springer-Verlag GmbH Germany, part of Springer Nature 2019

\begin{abstract}
Purpose Tracheostomy is usually suggested to facilitate airway management of intensive care unit (ICU) patients requiring prolonged translaryngeal intubation (PTLI). While it is not uncommon for physicians to hesitate and delay to perform it for more than 2 weeks, clinically recognizable airway adverse effects following PTLI are rarely discussed. Therefore, we compared retrospectively the PTLI group with control to assess them in adult patients.

Methods During a period of 1991-2012, patients aged older than 15 years that were admitted to University of Tsukuba Hospital ICU, underwent translaryngeal intubation (TLI) for 14 days or longer, were retrospectively studied as Group P. Patients whose tracheas were intubated for 13 days or less were set up as a control group (Group C). Patients were excluded if they had undergone any procedures that might have affected recurrent laryngeal nerves.

Results Ninety-eight patients ( $\mathrm{M}: \mathrm{F}=58: 40$ ) (group $\mathrm{P}$ ) and 88 patients ( $\mathrm{M}: \mathrm{F}=58: 30$ ) (group C) were included. There were no differences in patients' characteristics. Durations of TLI were $20.8 \pm 6.8$ days in group P and $3.8 \pm 3.0$ days in group C. There were no differences in the occurrence rates of severe airway adverse events. Although we found higher incidence rates of dysphagia and dysphonia/hoarseness in group P, the symptoms were mild and they were not prolonged. There were no differences in other signs and symptoms.

Conclusions We found no difference in the occurrence rates of severe airway adverse events in both groups. Translaryngeal intubation may be tolerable in adults even if the duration exceeds 2 weeks.
\end{abstract}

Keywords Prolonged translaryngeal intubation - Airway complications $\cdot$ Mechanical ventilation $\cdot$ Adult patients Retrospective observational study

\section{Introduction}

Translaryngeal intubation (TLI) is commonly performed for mechanical ventilation during anesthetic and intensive care settings as well as cardiopulmonary resuscitation. In the current practice, TLI is performed for 2 weeks or even longer in adults $[1,2]$, while TLI is kept for much longer

Takeru Shimizu

taksmz@md.tsukuba.ac.jp

1 Department of Anaesthesiology, Faculty of Medicine, University of Tsukuba, 1-1-1 Tennodai, Tsukuba 305-8575, Ibaraki, Japan

2 Department of Emergency and Critical Care Medicine, Faculty of Medicine, University of Tsukuba, 1-1-1 Tennodai, Tsukuba 305-8575, Ibaraki, Japan

3 Department of Anaesthesia, Ibaraki Prefectural Central Hospital, 6528 Koibuchi, Kasama 309-1793, Ibaraki, Japan periods in children [3]. As many as $30 \%$ of patients requiring prolonged mechanical ventilation (MV) eventually receive a tracheotomy [4]. When prolonged MV is required, a tracheostomy is usually suggested to facilitate airway management in intensive care unit (ICU) [5]. On one hand, tracheostomy helps patients' well-being by reducing laryngeal irritation, lowering respiratory tract resistance, improving pulmonary toilet, enhancing patient communication, and decreasing the requirements for sedation [6]. On the other hand, this procedure is associated with complications including hemorrhage, site infections, pneumomediastinum, pneumothorax, tracheomalacia, tracheoesophageal fistula, and catastrophic arterial fistula [7-11]. Although some advocate early (within 4 days) tracheostomy [12], the optimal timing of tracheostomy is yet controversial $[13,14]$.

Many prospective studies have been published addressing the issue of tracheostomy and airway damage [7-11]. As it stands, a significant portion is dedicated to describing 
the timing of tracheostomy. However, patients' conditions may preclude performing tracheostomy in some cases, including severe bleeding tendency or infectious lesion in the anterior neck. Although we have so far encountered quite a few cases requiring PTLI in such conditions, we felt that severe complications had not been often observed. Therefore, we conducted this study to elucidate the occurrence of airway complications after PTLI.

The primary aim of this study was to compare retrospectively the clinically recognizable airway adverse effects in adult patients who underwent TLI for 14 days or longer to those who underwent TLI for less than 14 days.

\section{Methods}

\section{Patients}

This was a retrospective observational study in the mixed ICU of a university hospital. This study was approved by the University of Tsukuba Hospital ethics committee (No. 15-56). Patients aged older than 15 years were admitted to University of Tsukuba Hospital ICU during a period of January 1991-December 2012, whose tracheas were only intubated translaryngeally for 14 days or longer, whose tracheas were not tracheostomized at the time of extubation, and who were alive at ICU discharge were included in this study as PTLI group (group P). We also set up a non-PTLI group as a control group (group C). This group included patients on the ICU admission list whose tracheas were intubated for 13 days or less, and whose tracheas were not tracheostomized at extubation. Group $\mathrm{C}$ patients were selected from the same period of patients in group $\mathrm{P}$ so that the airway management manners were adjusted to be the same. Patients were not selected if they had undergone thoracic or cardiac surgery, pharyngeal and/or laryngeal surgery, thyroid surgery, or any procedures that might have affected recurrent laryngeal nerves. Patients whose tracheas were intubated nasally were also excluded.

\section{Data collection}

Patients' demographic features, type of admission (medical, scheduled surgery, and unscheduled surgery), intubation period, intervention of airway after extubation, signs and symptoms of laryngeal and tracheal complications, ICU-acquired pneumonia, and mortality within 27 days after the studied intubation period were recorded from the medical records.

\section{Data analysis}

Regarding the cases in which fiberscopic examination was not done, signs and symptoms were assessed using fourcategory grades based on clinical severity reported by Colice et al., i.e., (1) none, (2) mild; hoarseness lasting less than 12 weeks, designated as transient, (3) moderate; hoarseness lasting more than 12 weeks, designated as chronic, or post-extubation stridor not requiring tracheostomy, and (4) severe; post-extubation stridor requiring tracheostomy or aspiration [15].

\section{Statistical analysis}

Data are shown as the mean \pm SD for the data that were normally distributed. We compared demographic, clinical and peri-operative frequencies using $\chi^{2}$ and unpaired two-tailed $t$ tests with or without Welch's correction. For statistical comparisons between the groups, Fisher's exact test was used. GraphPad Prism version 6 (GraphPad Software, San Diego, CA, USA) was used for statistical analysis. A $p$ value less than 0.05 was considered to be significant.

\section{Results}

During the period from January 1991 to December 2012, a total of 8388 patients were admitted to the multidisciplinary ICU, and 6320 patients were age over 15 years. Mechanical ventilation (MV) was applied to 3347 patients in total of age over 15 years, and 263 patients were on MV for more than 14 days. In $98(\mathrm{M}: \mathrm{F}=58: 40)$ patients, TLI was used solely during $\mathrm{MV}$, and they were enrolled in the group $\mathrm{P}$ (Fig. 1). On the other hand, group $\mathrm{C}$ consisted of 88 patients $(\mathrm{M}: \mathrm{F}=58: 30)$. There was no significant difference between the two groups in terms of demographics [mean age $59 \pm 17$ (range 15-90) in group P and 60 15 (range, 18-81) in group C]. Concerning neurological patients, there was no difference in this criterion (10 in group $\mathrm{C}$, and 4 in group $\mathrm{P})$. However, more patients for scheduled surgeries were included in the control group, and more patients for unscheduled surgeries were included in the group P (Table 1). The last patient included in group $\mathrm{T}$ appeared in 2010.

Outcome measures of the patients are shown in Table 2. Other than intubation period, there were no differences between the groups, including tracheotomy performed within 7 days after extubation, and mortality within 27 days after extubation. Pneumonia, including ventilator-associated pneumonia (VAP) and others, was not newly developed in either of the groups. Respiratory signs and symptoms were observed at the time of extubation in some of the cases 


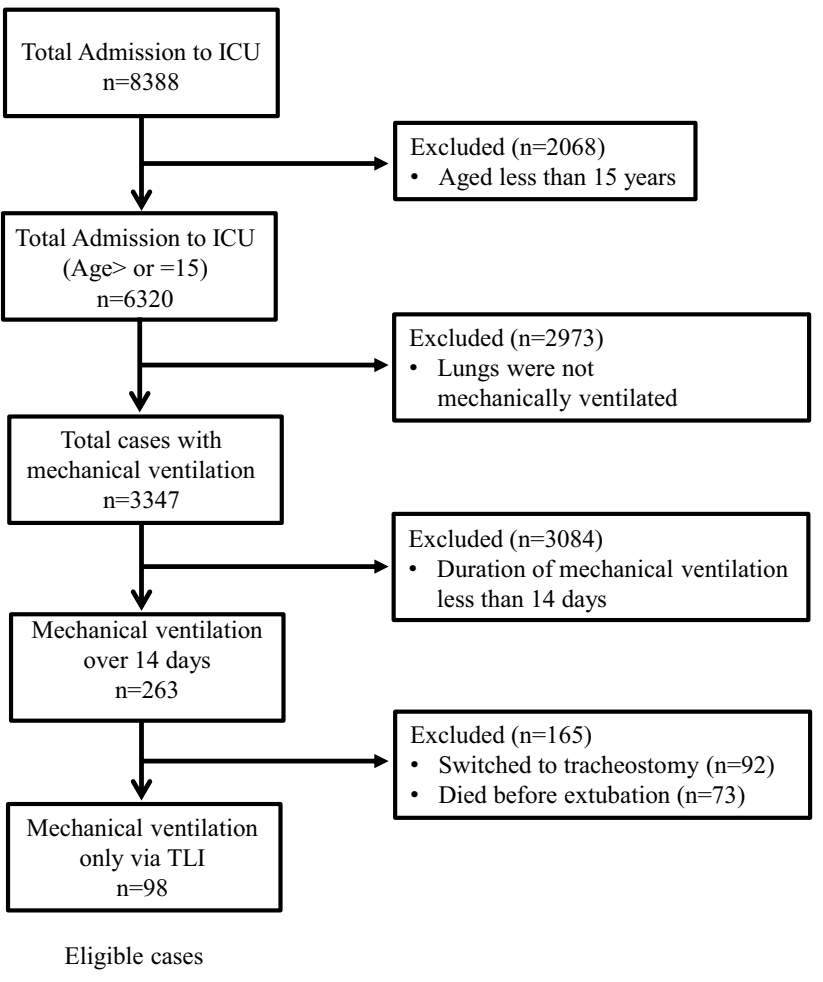

Fig. 1 Patient flow chart for prolonged translaryngeal intubation

(Table 3). However, these were the remaining signs and/or symptoms of pneumonia, including purulent sputum, rales on auscultation, and infiltrates on chest X-ray, which were the original causes of intubation.

The rates of the patients who had at least one sign and/ or symptom were not significantly different (42\% in group $\mathrm{C}$ versus 52\% in group $\mathrm{P}$ ). Occurrence rate of signs and symptoms was not different between the groups except for dysphagia and dysphonia/hoarseness (Table 3). In terms of these symptoms, not only the occurrence rate, but severity of these symptoms were also higher in group $\mathrm{P}$ compared to group C (Table 4). Yet, most of the patients who had signs and/or symptoms were classified as mild, namely they did not last more than 12 weeks. Laryngeal granuloma was observed in three patients in total, but no surgical interventions were required in these cases.

\section{Discussion}

This is the first report comparing outcomes of short-term and prolonged translaryngeal intubation $>14$ days in patients admitted to ICU. Tracheostomy and TLI have their own benefits and drawbacks. Major advantages of tracheostomy compared to TLI are described as improved patient comfort, less need for sedation, lower work of breathing, improved patient safety, improved oral hygiene, and so on [8]. However, patients' conditions may preclude performing tracheostomy in certain cases, including severe bleeding conditions, recent sternotomy, and, infectious lesions in anterior neck. If patients are with these conditions together with difficulties in early prediction of eventual need of tracheostomy, it is not uncommon for physicians to hesitate and delay to perform it for more than 2 weeks [16], where translaryngeal intubation is practically kept for 2 weeks [17]. It has been reported that translaryngeal intubation can be performed without significant increase of complications for up to 2 weeks [18]. The samples we could extract started from 1991, where physicians supposedly referred these reports. Therefore, we chose this duration for the cutoff. The reasons why tracheostomy was delayed could not be studied thoroughly because of the character of the study. Identified reasons were as follows.
Table 1 Characteristics of screened patients

\begin{tabular}{lllr}
\hline & Control & Prolonged TLI & $p$ values \\
\hline Sex (m:f) & $58: 30$ & $58: 40$ & 0.347 \\
Age (year) ${ }^{\mathrm{a}}$ & $60.3 \pm 15.1(63,18-81)$ & $58.5 \pm 16.9(62,15-90)$ & 0.453 \\
Weight $(\mathrm{kg})$ & $55.7 \pm 10.8$ & $59.7 \pm 16.4$ & 0.055 \\
Height $(\mathrm{cm})$ & $158.3 \pm 9.5$ & $159.7 \pm 9.8$ & 0.376 \\
BMI & $22.3 \pm 3.8$ & $23.1 \pm 6.1$ & 0.270 \\
Type of admission $(n)$ & & & \\
Medical & 30 & 40 & 0.367 \\
Scheduled surgery* & 55 & 34 & 0.0002 \\
Unscheduled surgery* & 3 & 24 & $<0.0001$ \\
Neurological patients $(n)$ & 10 & 4 & 0.071 \\
Medical & 7 & 4 & 0.227 \\
Surgical & 3 & 0 & 0.059 \\
\hline
\end{tabular}

Other values are given as mean $\pm \mathrm{SD}$

$* p<0.05$

${ }^{\mathrm{a}}$ Values are expressed as mean $\pm \mathrm{SD}$ (median, range) 
Table 2 Outcome measures

\begin{tabular}{lllc}
\hline & Control & Prolonged TLI & $p$ values \\
\hline Intubation period (days)* & $3.8 \pm 3.0(2,2-6$ & $20.8 \pm 6.8(18,15-24$ & $<0.0001$ \\
& $[1-13])$ & $[14-50])$ & \\
Tracheostomy within 7 days after extubation & 4 & 6 & $>0.9999$ \\
Reintubation within 7 days after extubation & 2 & 2 & $>0.9999$ \\
Immediate reintubation & 2 & 0 & 0.223 \\
Symptoms of pneumonia (remaining) & 4 & 3 & 0.709 \\
Mortality within 27 days & 3 & 4 & $>0.9999$ \\
\hline
\end{tabular}

*Values are expressed as mean $\pm \mathrm{SD}$ (median, IQR [range])

Table 3 Airway assessment

\begin{tabular}{lccc}
\hline & Control & Prolonged TLI & $p$ values \\
\hline Assessment with fiberscopy & 3 & 17 & 0.004 \\
Assessment only by clinical manifestations & 85 & 81 & 0.0086 \\
At least one sing and/or symptom existed & 33 & 51 & 0.056 \\
Dysphagia & 7 & 26 & 0.001 \\
Dysphonia/hoarseness & 5 & 18 & 0.013 \\
Difficulty in expectoration & 23 & 37 & 0.116 \\
Abnormal vocal cord mobility & 6 & 9 & 0.416 \\
Laryngeal edema & 1 & 7 & 0.068 \\
Post-intubation laryngeal granuloma & 2 & 1 & $>0.9999$ \\
\hline
\end{tabular}

Table 4 Severity of signs and symptoms

\begin{tabular}{llllll}
\hline & Severe & Moderate & Mild & None & $p$ values \\
\hline Dysphagia & $2 / 3$ & $0 / 0$ & $3 / 17$ & $55 / 40$ & 0.0216 \\
Dysphonia/ hoarseness & $0 / 1$ & $0 / 0$ & $4 / 12$ & $56 / 47$ & 0.0195 \\
Abnormal vocal cord mobility & $0 / 0$ & $0 / 0$ & $4 / 4$ & $56 / 56$ & 1.00 \\
Laryngeal edema/ vocal cord swelling & $0 / 0$ & $0 / 0$ & $1 / 4$ & $59 / 56$ & 0.1742 \\
\hline
\end{tabular}

Values on left side are for control group and right side for prolonged TLI group
Localized neck infection and coagulopathies seemed to be the main pathologies. In addition, obesity, and the thick fat tissues around the neck discouraged physicians to perform a tracheostomy. In some cases, physicians planned to extubate the patients' tracheas, but hesitated and suspended it ending up with day-to-day observation. In other cases, families did not accept the procedures.

For the airway assessment, we focused on the major signs and symptoms as shown in Table 4, which could be extracted from the patients' medical records. At the same time, since these signs and symptoms were also discussed and classified before [15], they can be generalized for the severity assessment. We found that there were higher occurrences of dysphagia and dysphonia/hoarseness in group P, and that the severity of these symptoms was also higher in group P. Most of the cases that had significantly more occurrence compared to group $\mathrm{C}$ were classified as mild. There were three severe cases with dysphagia in group P. However, we found that even 1-3 days of TLI also caused two severe dysphagia cases, which are comparative to group P. Although these results would rather indicate that the shorter TLI had relatively high incidence of these events, it seems these were even smaller than reported values [15].

Griffiths et al. reported in the meta-analysis that performing a tracheostomy at an earlier stage than is currently practiced may shorten the duration of artificial ventilation and length of ICU stay [19]. However, because of the characteristic of our study design, we were unable to evaluate whether or not PTLI had prolonged the duration of mechanical ventilation compared to patients with tracheostomy.

We did not observe VAP in both groups, where reported incidences per 1000 ventilator days were 1.4 in Japan in 2014 [20] and 0.0-4.4 in the US in 2012 [21]. This is compatible with the previous report that the incidence of pneumonia is the same in tracheostomy and TLI groups [22]. However, selection bias may exist in the evaluation of VAP. The data could be interpreted that those patients selected as group P were kept on TLI rather than having been tracheostomised 
just because they did not have pneumonia. In other words, those who had infection, increase of secretion, or VAP might have been tracheostomised soon after tracheal intubation. Therefore, we cannot conclude that PTLI may not increase or cause VAP regardless of the sample size.

This study has several limitations. First, this is a retrospective and single-center study evaluating patients who underwent mechanical ventilation, over the period of 22 years. Yet, we found only 98 samples for PTLI in total, which affects to draw the general conclusions. It is likely that numerous aspects of care changed over this period of study making this a heterogeneous population from which it is difficult to draw conclusion [12]. Airway management in mechanically ventilated patients might be slightly different from hospital to hospital in details, especially sedation during mechanical ventilation [23]. Patients treated earlier in the study period commonly received relatively deep sedation due to poor ventilator capability of synchronization with patients' spontaneous breathing. Therefore, we need to examine whether or not the present results can be applied to other institutions cautiously. Second, this is not a study to compare the clinical outcomes following early tracheostomy or PTLI. We compared the short-term TLI and prolonged TLI. Therefore, it is unclear whether PTLI worsened the clinical measures. In other words, our study cannot refer whether the duration of mechanical ventilation was shortened if tracheostomy had been done. Recent two meta-analyses may support this concern $[24,25]$. The authors found no evidence that early tracheostomy reduced mortality (both short term and long term), duration of mechanical ventilation, ICU stay, or VAP, except for a shorter duration of sedation. However, in the present study, the sample size might be too small to conclude that there was no significant difference in terms of the above variables. Third, we did not perform fiberoptic examinations in all of the cases. Only 20 cases out of 186 cases (10.8\%) were confirmed objectively. Therefore, there is a possibility to underestimate the tissue or physical damages just because of the absence of signs and symptoms. Fourth, we did not match study subjects using propensity scores due to following reasons. According to the general comprehension, patients with same propensity scores are supposed to receive therapeutic options randomly. However, in the current study, the control cases were chosen from patients who had not undergone surgeries possibly affecting laryngeal functions, namely it was not a random sampling. In addition, the proportion of PTLI was very small (1.2\%) among the patients admitted to the ICU (or even $2.9 \%$ of patients on MV), and propensity scores would not predict the therapeutic options in this setting. In the first place, PTLI was not elective or chosen intentionally. Fifth, we could not specify the difficulty scores, including Mallampati score, and Cormack-Lehane classification, because these evaluations were not fully recorded in the patients' charts.
In conclusion, we found no differences in the occurrence rate of severe airway adverse events even if airway management was done only by translaryngeal intubation. Although we found higher incidence rates of dysphagia and dysphonia/ hoarseness in group $\mathrm{P}$, the symptoms were mild and they were not prolonged in most of the cases. Our study suggests that TLI may be tolerable in adult patients even if the duration exceeds 2 weeks.

Acknowledgements This research was solely supported by departmental resources.

\section{Compliance with ethical standards}

Conflict of interest The authors declared no conflicts of interest and received no financial support for the research, authorship, and/or publication of this article.

Ethical approval This study was approved by the institutional ethics committee (No. 15-56).

\section{References}

1. Kluge S, Baumann HJ, Maier C et al (2008) Tracheostomy in the intensive care unit: a nationwide survey. Anesth Analg 107:1639-1643

2. Blot F, Melot C; Commission d'Epidémiologie et de Recherche Clinique. Indications, timing, and techniques of tracheostomy in 152 French ICUs. Chest 2005; 127: 1347-1352.

3. Zaima A, Bitoh Y, Morita K, Tsugawa J, Ishii T, Satoh S, Nishijima E (2010) Long-term T-tube stenting as definitive treatment of severe acquired subglottic stenosis in children. J Pediatr Surg 45:996-999

4. Combes A, Luyt CE, Nieszkowska A, Trouillet JL, Gibert C, Chastre J (2007) Is tracheostomy associated with better outcomes for patients requiring long-term mechanical ventilation? Crit Care Med 35:802-807

5. Durbin CG Jr (2010) Tracheostomy: why, when, and how? Respir Care 55:1056-1068

6. Nieszkowska A, Combes A, Luyt CE, Ksibi H, Trouillet JL, Gibert C, Chastre J (2005) Impact of tracheotomy on sedative administration, sedation level, and comfort of mechanically ventilated intensive care unit patients. Crit Care Med 33:2527-2533

7. Needham DM, Bronskill SE, Sibbald WJ, Pronovost PJ, Laupacis A (2004) Mechanical ventilation in Ontario, 1992-2000: incidence, survival, and hospital bed utilization of noncardiac surgery adult patients. Crit Care Med 32:1504-1509

8. Durbin CG Jr (2005) Early complications of tracheostomy. Respir Care 50:511-515

9. Epstein SK (2005) Late complications of tracheostomy. Respir Care 50:542-549

10. Scales DC, Guan J, Martin CM, Redelmeier DA (2006) Administrative data accurately identified intensive care unit admissions in Ontario. J Clin Epidemiol 59:802-807

11. Scales DC, Thiruchelvam D, Kiss A, Redelmeier DA (2008) The effect of tracheostomy timing during critical illness on long-term survival. Crit Care Med 36:2547-2557

12. Young D, Harrison DA, Cuthbertson BH, Rowan K (2013) Effect of early vs late tracheostomy placement on survival in patients 
receiving mechanical ventilation: the TracMan Randamized Trial. JAMA 309:2121-2129

13. Macintyre NR (2001) Evidence-based guidelines for weaning and discontinuing ventilator support: a collective task force facilitated by the American College of Chest Physicians; the American Association for Respiratory Care; and the American College of Critical Care Medicine. Chest 120:375S-S396

14. Cheung NH, Napolitano LM (2014) Tracheostomy: epidemiology, indications, timing, technique, and outcomes. Respir Care 59:895-915

15. Colice GL, Stukel TA, Dain B (1989) Laryngeal complications of prolonged intubation. Chest 96:877-884

16. Park YS, Lee J, Lee SM, Yim JJ, Kim YW, Han SK, Yoo CG (2012) Factors determining the timing of tracheostomy in medical ICU of a tertiary referral hospital. Tuberc Respir Dis (Seoul) 72: 481-485

17. Maziak DE, Meade MO, Todd TR (1998) The timing of tracheotomy: a systematic review. Chest 114:605-609

18. Dunham CM, LaMonica C (1984) Prolonged tracheal intubation in the trauma patient. J Trauma 24:120-124

19. Griffiths J, Barber VS, Morgan L, Young JD (2005) Systematic review and meta-analysis of studies of the timing of tracheostomy in adult patients undergoing artificial ventilation. BMJ 330:1243-1247

20. Japan Nosocomial Infections Surveillance (JANIS), Ministry of Health, Labor, and Welfare (2017). https://janis.mhlw.go.jp/repor
t/open_report/2017/3/3/ICU_Open_Report_201700.pdf. Accessed 30 May 2019

21. Dudeck MA, Weiner LM, Allen-Bridson K, Gross C, Malpiedi PJ, Peterson KD et al (2013) National Healthcare Safety Network (NHSN) report, data summary for 2012, device-associated module. Am J Infec Control 41:1148-1166

22. Shimi NS, Mebazaa M, Mestiri T, Ben Ammar MS (2004) Tracheotomy or prolonged tracheal intubation for long-term ventilation. Tunis Med 82:805-808

23. Raede MC, Finfer S (2014) Sedation and delirium in the intensive care unit. N Engl J Med 370:444-454

24. Huang H, Li Y, Adriani F, Chen X, Lin J (2014) Timing of tracheostomy in critically ill patients: a meta-analysis. PLoS ONE 9:e92981

25. Szakmany T, Russell P, Wilkes AR, Hall JE (2015) Effects of early tracheostomy on resource utilization and clinical outcomes in critically ill patients: meta-analysis of randomized controlled trials. Br J Anaesth 114:396-405

Publisher's Note Springer Nature remains neutral with regard to jurisdictional claims in published maps and institutional affiliations. 